\title{
MAXIMUM ENTROPY APPROACH TO FUZZY CONTROL
}

\section{Arthur Ramer*, Vladik Kreinovich"*}

- School of CSE, University of New South Wales, P.O. Box 1, Kensington, N,S.W. 2099, Australia

- Computer Science Department, University of Tezas at El Paso, El Paso, TX 79968, vladik@cs.ep.utexas.edu

\section{ABSTRACT}

For the same expert knowledge, if we use different \& - and $V$-operations in a fuzzy control methodology, we end up with different control strategies. Each choice of these operations restricts the set of possible control strategies. Since a wrong choice can lead to a low quality control, it is reasonable to try to loose as few possibilities as possible. We formalize this idea and show that it leads to the choice of $\min (a+b, 1)$ for $V$ and $\min (a, b)$ for $\&$. This choice was tried on NASA Shuttle simulator; it leads to a maximally stable control.

\section{INTRODUCTION, MOTIVATIONS, AND MAIN RESULTS}

In rifany situations (e.g., in space exploration), it is necessary to translate operator's knowledge )(formulated in natural-language terms) into actual control. According to a fuzzy control methodology [1, 10, 11], we must, first, determine membership functions that correspond to all natural- language terms (like "small" or "big") that appear in the rules. Second, we must choose operations that correspond to \& and $V$. As a result we get a membership function $\pi_{C}(u)$ for a control; then we need a method to transform this function $\pi_{C}(u)$

Different choices of \& - and $V$ - operations lead to control strategies of drastically different quality, so the

1) When we make a choice, we thus restrict the set of possible control strategies. Since a wrong choice can lead to a low quality control, it sounds reasonable to try to loose as few possibilities as possible. In other words, it sounds reasonable to choose $\&$ - and $V$-operations in such a way that uncertainty corresponding to $\pi_{C}(u)$ is the biggest possible. This methodology is well known in the case when the uncertainty is probabilistic; it is called a maximum entropy approach, and it is widely applied to various problems ranging from processing physical data to processing uncertainties in expert systems $[2,4,6,7]$.

Just like in a probabilistic case, we want to evaluate the uncertainty of a membership function as the average number of binary questions that one needs to ask in order to determine the value. We prove that the desired maximal uncertainty is attained when we use $\min (a+b, 1)$ for $V$, and $\min (a, b)$ for \&. In control terms, this maximum entropy approach is proved to lead to maximally stable controls. This result is in good accordance with common sense: we minimized the lost opportunities, and therefore, we ended up with the best possible control. It is also in good accordance with the experiments on the NASA Johnson Space Center Shuttle silumator, that showed that this choice of $\&$ - and $V$-operations lead to a maximally stable control $[8,9]$.

2) The above arguments are reasonable only if we are ready to apply various defuzzification techniques to extract the best control from $\pi_{C}(u)$. However, in industrial applications, a defuzzification rule is usually fixed. Since this rule is not necessarily the most appropriate (see., e.g., $[9,14]$ ), it is reasonable to try to depend on it to a smallest extent. In other words, in these cases, it is reasonable to choose \& and Voperations from the condition that the uncertainty related to $\pi_{C}(u)$ is the smallest possible.

We prove that this leads to the choice of $\max (a, b)$ for $V$ and $a b$ for \&. In control terms, this minimum entropy approach leads to maximally smooth controls. This result is also in good corresponds with common sense: since we were extremely cautious, we ended up with a very smooth control. This result is also in good 
accordance with the experiments on the NASA Space Shuttle simulator, that show that this kind of control is the smoothest, and hence, it is the best control for problems like docking where smoothness is the main requirement.

Detailed description and proofs can be found in [3].

\section{HOW TO MEASURE UNCERTAINTY THAT CORRESPONDS TO A MEMBERSHIP FUNCTION}

In order to answer this question, let us recall where the values $\pi(x)$ of a membership function come from. If $\pi(x)$ corresponds to, say, "small", then $\pi(x)$ is our degree of belief that $x$ is small. One of the most natural ways to express this "degree of belief" by a number is to ask several experts, whether they consider $x$ small or not, and after $M$ out of $N$ answer "yes", take $M / N$ as $\pi(x)$ (see, e.g., [5]). This approach allows us to interpret the value $\pi(x)$ as a frequency (or, if you like, subjective probability) that $x$ is small.

Suppose that we have a notion (like "small") that is described by a function $\pi(x)$. If the only thing we know about some real value $x$ is that it satisfies this property (e.g., "is small"), then how many binary questions do we have to ask to determine $x$ ?

1. If we have finitely many (say $N$ ) alternatives, then it is natural to estimate the uncertainty by the smallest number $Q(N)$ of binary ("yes" - "no") that we have to ask in order to determine the alternative. It is well known that this smallest number is attained when we apply binary search, and $Q(N) \approx \log _{2}(N)$.

2. If the only knowledge we have is that the unknown value $x$ belongs to an interval $[a, b]$, then for each $\varepsilon>0$, we can define the $\varepsilon$-uncertainty $Q([a, b], \varepsilon)$ of this knowledge as the smallest number of binary questions that we have to ask in order to determine $x$ with precision $\varepsilon$ (i.e., in order to find an interval $\left[x_{0}-\varepsilon, x_{0}+\varepsilon\right]$ that contains $x$ ). For that, we can divide $[a, b]$ into $\approx(b-a) /(2 \varepsilon)$ intervals $[a, a+2 \varepsilon],[a+2 \varepsilon, a+4 \varepsilon], \ldots$, and thus $Q([a, b], \varepsilon) \approx \log _{2}((b-a) /(2 \varepsilon)) \sim u-m \log _{2}(2 \varepsilon)$, where $u=\log _{2}(b-a)$ and $m=1$.

3. If the only knowledge we have is that $x$ belongs to a finite union $S$ of intervals, then we get a similar formula with $u=\log _{2}(\mu(S))$, where $\mu(S)$ is the total length (measure) of $S$.

4. Let us now consider the case when our knowledge about $x$ is described by a piecewise-constant membership function, i.e., there exist values $x_{1}<x_{2}<\ldots<x_{n}$ such that $\pi(x)=0$ for $x<x_{1}$ and $x>x_{n}, \pi(x)=$ const on each of the intervals $\left(x_{i}, x_{i+1}\right)$, and for each $i, \pi\left(x_{i}\right)$ coincides either with the value of $\pi(x)$ for $x<x_{i}$, or with the values of $\pi(x)$ for $x>x_{i}$. Such a function takes takes only finitely many different values $h_{0}=0<h_{1}<h_{2}<\ldots<h_{k}$.

So, $h_{1}$-th part of th experts believe that $x$ belongs to the set $\left\{x: \pi(x) \geq h_{1}\right\},\left(h_{2}-h_{1}\right)$ of them believe that $x \in\left\{x: \pi(x) \geq h_{2}\right\},\left(h_{3}-h_{2}\right)$ of them believe that $x \in\left\{x: \pi(x) \geq h_{3}\right\}, \ldots$, and $h_{k}-h_{k-1}$ of them believe that $x \in\left\{x: \pi(x) \geq h_{k}\right\}$. If $x \in\left\{x: \pi(x) \geq h_{1}\right\}$, then we need $Q\left(\left\{x: \pi(x) \geq h_{1}\right\}, \varepsilon\right)$ questions to determine $x$ with the precision $\varepsilon$. If $x \in\left\{x: \pi(x) \geq h_{2}\right\}$, then we need $Q\left(\left\{x: \pi(x) \geq h_{2}\right\}, \varepsilon\right)$ questions, etc. Therefore, according to the opinion of $h_{1}$ of experts, we need $Q\left(\left\{x: \pi(x) \geq h_{1}\right\}, \varepsilon\right)$ questions; according to the opinion of $\left(h_{2}-h_{1}\right)$ of the experts, we need to ask $Q\left(\left\{x: \pi(x) \geq h_{2}\right\}, \varepsilon\right)$ questions, etc. So, it is natural to define the expected number of questions as $Q(\pi, \varepsilon)=\sum_{i=0}^{k-1}\left(h_{i+1}-h_{i}\right) Q\left(\pi_{\left\{x: \pi(x) \geq h_{i+1}\right\}}, \varepsilon\right)$. One can prove that in this case, $m=\sup _{x} \mu(x)$ and $u=\sum_{i=0}^{k-1}\left(h_{i+1}-h_{i}\right) \log _{2}\left(\mu\left\{x: \pi(x) \geq h_{i+1}\right\}\right)$.

5. An arbitrary continuous membership $\pi$ function can be approximated by piecewise-constant functions $\pi_{n}$. So, we can define its $\varepsilon$-uncertainty $Q(\pi, \varepsilon)$ as the limit of the values $Q\left(\pi_{n}, \varepsilon\right)$. One can prove that $Q(\pi, \varepsilon) \sim m(\pi)-u(\pi) \log _{2}(2 \varepsilon)$, where $m(\pi)=\sup _{x} \pi(x)$, and $u(\pi)=\int_{0}^{m(x)} \log _{2}(\mu(\{x: \pi(x) \geq h\})) d h$. In particular, if for some $x_{0}>0, \pi(x)=0$ for $x<0, \pi(0)=1, \pi(x)=0$ for $x \geq x_{0}$, and for $x \in\left[0, x_{0}\right), \pi(x)$ is continuous and decreasing, then $u(\pi)=\log _{2}\left(x_{0}\right)-(1 / \ln (2)) I(\pi)$, where $I(\pi)=\int_{0}^{x_{0}}(1-\pi(x)) / x d x$ coincides with the expression that was introduced (from different assumptions) in $[12,13]$.

How to compare uncertainties of different membership functions? We say that uncertainty $\left(u_{1}, m_{1}\right)$ is not smaller than the uncertainty $\left(u_{2}, m_{2}\right)$, and denote it by $\left(u_{1}, m_{1}\right) \succeq\left(u_{2}, m_{2}\right)$ if there exists an $\varepsilon_{0}$ such that for all $\varepsilon<\varepsilon_{0}, u_{1}-m_{1} \log _{2}(2 \varepsilon)>u_{2}-m_{2} \log _{2}(2 \varepsilon)$. One can prove that $\left(u_{1}, m_{1}\right) \succeq\left(u_{2}, m_{2}\right)$ if and only if either $m_{1}>m_{2}$, or $m_{1}=m_{2}$ and $u_{1} \geq u_{2}$.

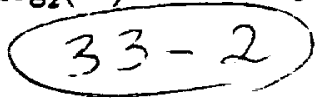




\section{WHAT IS FUZZY CONTROL? A BRIEF MATHEMATICAL EXPLANATION}

Definition 1. Assume that a set $S \subset R^{n}$ is given. Elements $\vec{x}=\left(x_{1}, \ldots, x_{n}\right) \in S$ will be called states.

Comment. Informally, the values $x_{1}, \ldots, x_{n}$ describe everything that we need to know to make a control decision. For example, if we control a heater/cooler, then $n=1$, and the only variable we need to know is the difference $x_{1}=t-t_{0}$ between the actual and the desired temperature. If we are controlling a spaceship, then we need to know its coordinates $x_{1}, x_{2}, x_{3}$, its current velocity vector ( 3 more variables $\left.x_{4}=\dot{x}_{1}, x_{5}=\dot{x}_{2}, x_{6}=\dot{x}_{3}\right)$, and 2 angles that describe the orientation. So, for a spaceship, $n=8$.

Definition 2. Let us fix a finite set $\mathcal{P}$ of continuous membership functions. The elements of this set will be called fuzzy properties.

Comment. As examples, we can consider "small", "big", "medium", etc.

Definition 3. By an elementary formula $E$ we mean an expression of the type $P_{i}\left(x_{i}\right)$, where $P_{i}$ is a fuzzy property. By a rule, we mean an expression of the type $E_{1}, \ldots, E_{m} \rightarrow P(u)$, where $E_{i}$ are elementary formulas, $P$ is a fuzzy property, and $u$ is a special variable reserved for control. Formulas $E_{i}$ are called conditions, $P(u)$ is called a conclusion of the rule. By a knowledge base we mean a finite set of rules.

Comment. As an example of the rule, one can consider a rule $N\left(x_{1}\right) \rightarrow N(u)$, meaning that if the difference $t-t_{0}$ between the actual and the desired temperatures is negligible, then the control should be negligible. Another possible rule is: $S P\left(x_{1}\right) \rightarrow S N(u)$, meaning that if the difference $t-t_{0}$ is small positive, then we need to, apply a small negative control (i.e., switch on the cooler a little bit). A similar rule $S N\left(x_{1}\right) \rightarrow S P(u)$ tells that if it becomes a little bit cold, it is necessary to switch on the heater for a while.

Motivation of the following definitions. If we have a set of rules, then we can say that a control $u$ is appropriate if and only if one the rules is applicable, and $u$ appropriate according to this rule. Let us denote the statement "control $u$ is appropriate" by $C(u)$. Then, for the three rules that describe the cooler/heater, we have the following informal "formula" that describes when a control $u$ is appropriate:

$$
C(u) \equiv(N(x) \& N(u)) \vee(S P(x) \& S N(u)) \vee(S N(x) \& S P(u)) \text {. }
$$

Since $N(x), N(u), \ldots$, are fuzzy statements, we can get only fuzzy conclusions about the control, i.e., thus defined $C(u)$ also becomes a fuzzy statement. To get the precise values, we need to choose some operations that would describe $\&$ and $\vee$ for fuzzy values.

After we choose them, we can use the above formula to describe for each $u$, what is the reasonable degree of belief that this value $u$ is an appropriate control. In other words, we will be able to generate a membership function $\pi_{C}(u)$ that corresponds to control. After that, we need some defuzzification procedure that would transform this membership function into a single recommended control value.

The meaning of the $\&$-operation is as follows: Suppose that we have two statements $A$ and $B$. Our degree of belief in $A$ is equal to $a$, and our degree of belief in $B$ is equal to $b$. If we have no other information about $A$ and $B$, what must the reasonable degree of belief in $A \& B$ equal to? This reasonable degree of belief will be denoted by $f_{\&}(a, b)$. In the same situation, a reasonable degree of believe in $A \vee B$ will be denoted by $f_{V}(a, b)$, and $f_{V}$ will be called an $\vee$-operation.

In describing uncertainty of a membership function, we used the interpretation of membership values $\pi(x)$ as frequencies. Namely, we assumed that as a truth value $t(A)$ of an uncertain statement $A$, we take the ratio $t(A)=N(A) / N$, where $N(A)$ is the number of experts who believe in $A$, and $N$ is the total number of experts that were questioned. In this interpretation, the following inequalities are true: $N(A \vee B) \leq N(A)+N(B)$, $N(A \vee B) \leq N, N(A \vee B) \geq N(A)$ and $N(A \vee B) \geq N(B)$. If we divide both sides of these inequalities by $N$, and combine them into one, we get the following inequality: $\max (t(A), t(B)) \leq t(A \vee B) \leq \min (t(A)+t(B), 1)$, hence $\max (a, b) \leq f_{V}(a, b) \leq \min (a+b, 1)$. Likewise, from $N(A \& B) \leq N(A)$ and $N(A \& B) \leq N(B)$ we conclude that $t(\overline{A \& B}) \leq \min (t(A), t(B))$ and $f_{\&}(a, b) \leq \min (a, b)$.

If belief in $A$ and belief in $B$ were independent events, then we would have $t(A \& B)=t(A) t(B)$. In real life, beliefs are not independent: if an expert has strong beliefs in several statements that later turn out to be true, then this means that he is really a good expert, and therefore it is reasonable to expect that his degree

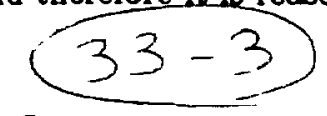


of belief in other statements that are true is bigger. If $A$ and $B$ are complicated statements, then many of those experts who believe in $A$ are really good experts, and therefore they believe in $B$ as well (and hence in $A \& B)$. Therefore, the total number $N(A \& B)$ of experts who believe in $A \& B$ must be bigger than the same number in the case when beliefs in $A$ and $B$ were uncorrelated random events. So $t(A \& B) \geq t(A) t(B)$, and $f_{\&}(a, b) \geq a b$. In statistical terms we can express this inequality by saying that $A$ and $B$ are non-negatively correlated. So, we arrive at the following definitions:

Definition 4[9]. By an and-or pair we will understand a pair of continuous functions $f_{k x}, f_{v}:[0,1] \times[0,1] \rightarrow$ $[0,1]$, that are non-decreasing in both variables, and satisfy the following conditions:

$\max (a, b) \leq f_{d}(a, b) \leq \min (a+b, 1) ; f_{V}(a, b) \leq \min (a, b) ; f_{\&}(0, a)=0, f_{\&}(1, a)=a, f_{V}(0, a)=a, f_{V}(1, a)=$ $1 ; f_{V}(a, b)=f_{V}(b, a), f_{\&}(a, b)=f_{\&}(b, a)$. An and-or pair is called correlated if $f_{\&}(a, b) \geq a b$ for all $a$ and $b$.

Denotation. For three numbers $a, b, c$, we define $f_{\&}(a, b, c)=f_{\&}\left(f_{\&}(a, b), c\right)$. For more than three numbers $a, b, \ldots, c$, we define $f_{k}(a, b, \ldots, c)=f_{\&}\left(\ldots\left(f_{k}\left(f_{k}(a, b), \ldots\right) c\right)\right.$. Likewise, we define $f_{v}(a, b, \ldots, c)=$ $f_{v}\left(\ldots\left(f_{v}\left(f_{v}(a, b), \ldots\right) c\right)\right.$.

Definition 5. Suppose that we are given a knowledge base $K=\left\{R_{1}, R_{2}, \ldots\right\}$, an and-or pair $\left(f_{\&}, f_{v}\right)$, and a state $\bar{x} \in S$. By a membership function, that corresponds to a rule $P_{1}\left(x_{i_{1}}\right), \ldots, P_{m}\left(x_{i_{m}}\right)-P(u)$, we mean a function $\pi_{R}=f_{\varepsilon}\left(P_{1}\left(x_{i_{2}}\right), \ldots, P_{m}\left(x_{i_{m}}, P(u)\right)\right.$. By a control membership function, that corresponds to the knowledge base and a state $\bar{x}$, we mean a function $\pi_{C}(u)=f_{V}\left(\pi_{R_{1}}, \pi_{R_{3}}, \ldots\right)$, where $R_{i}$ are rules from $K$.

\section{OPERATIONS FOR WHICH UNCERTAINTY IS THE BIGGEST}

Theorem 1. Suppose that $K$ is a knowledge base, and $\bar{x}$ is a state. Let us denote by $\pi_{C}(u)$ the control membership function, that corresponds to an arbitrary and-or pair $\left(f_{\&}(a, b), f_{V}(a, b)\right)$, and by $\bar{\pi}_{C}(u)$ the control membership function that corresponds to the and-or pair $(\min (a, b), \min (a+b, 1))$. Then, $\left(u\left(\bar{\pi}_{C}\right), m\left(\bar{\pi}_{C}\right)\right) \succeq\left(u\left(\pi_{C}\right), m\left(\pi_{C}\right)\right)$.

So, the biggest uncertainty is attained when we use $\min (a, b)$ for $\&$, and $\min (a+b, 1)$ for $V$. It has been proved in [9] that these very and-or operations lead to maximally stable controls. This result is in good accordance with common sense: we minimized the lost opportunities, and therefore, we ended up with the best possible control.

\section{OPERATIONS FOR WHICH UNCERTAINTY IS THE SMALLEST}

Theorem 2. Suppose that $K$ is a knowledge base, and $\bar{x}$ is a state. Let us denote by $\pi_{C}(u)$ the control membership function, that corresponds to an arbitrary correlated and-or pair $\left(f_{\&}(a, b), f_{v}(a, b)\right)$, and by $\tilde{\pi}_{C}(u)$ the control membership function that corresponds to the and-or pair $\left(a b, \max (a, b)\right.$. Then, $\left(u\left(\pi_{C}\right), m\left(\pi_{C}\right)\right) \succeq$ $\left(u\left(\bar{\pi}_{C}\right), m\left(\tilde{\pi}_{C}\right)\right)$.

So, the smallest uncertainty is attained when we use $a b$ for $\&$, and $\max (a, b)$ for $V$. It has been proved in [9] that these very and-or operations lead to maximally smooth controls. This result is also in good corresponds with common sense: since we were extremely cautious, we ended up with a very smooth control.

\section{PROOFS}

Lemma. If $\pi_{1}(x)$ and $\pi_{2}(x)$ are membership functions, and $\pi_{1}(x) \geq \pi_{2}(x)$ for all $x$, then $\left(u\left(\pi_{1}\right), m\left(\pi_{1}\right)\right) \succeq$ $\left(u\left(\pi_{2}\right), m\left(\pi_{2}\right)\right)$.

Proof of the Lemma. First, since $\pi_{1}(x) \geq \pi_{2}(x)$ for all $x$, we can conclude that $m\left(\pi_{1}\right)=\sup _{x} \pi_{1}(x) \geq$ $\sup _{x} \pi_{2}(x)=m\left(\pi_{2}\right)$. If $m\left(\pi_{1}\right)>m\left(\pi_{2}\right)$, then $\left(U\left(\pi_{1}\right), m\left(\pi_{1}\right)\right) \succeq\left(U\left(\pi_{2}\right), m\left(\pi_{2}\right)\right)$. So, to prove the Lemma, it remains to prove that if $m\left(\pi_{1}\right)=m\left(\pi_{2}\right)$, then $U\left(\pi_{1}\right) \geq U\left(\pi_{2}\right)$. Indeed, since we assumed that $\pi_{1}(x) \geq \pi_{2}(x)$, if $\pi_{2}(x) \geq h$, then $\pi_{1}(x) \geq h$. So, $\left\{x: \pi_{2}(x) \geq h\right\} \subset\left\{x: \pi_{1}(x) \geq h\right\}$. Therefore, for every $h, \mu\left(\left\{x: \pi_{1}(x) \geq\right.\right.$ $h\}) \geq \mu\left(\left\{x: \pi_{2}(x) \geq h\right\}\right)$. Since we assumed that $m\left(\pi_{1}\right)=\sup _{x} \pi_{1}(x)=\sup _{x} \pi_{2}(x)=m\left(\pi_{2}\right)$, we can integrate this inequality from 0 to $m\left(\pi_{i}\right)=\sup _{x} \pi_{i}(x)$, and conclude that $\int_{0}^{m\left(\pi_{1}\right)} \mu\left(\left\{x: \pi_{1}(x) \geq h\right\}\right) d h \geq$ $\int_{0}^{m\left(\pi_{2}\right)} \mu\left(\left\{x: \pi_{2}(x) \geq h\right\}\right) d h$, i.e., that $u\left(\pi_{1}\right) \geq u\left(\pi_{2}\right)$. Q.E.D.

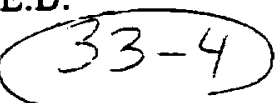


Proof of Theorem 1. According to Definition $5, \pi_{C}(u)=f_{V}\left(\pi_{R_{1}}, \pi_{R_{2}}, \ldots\right)$, where $R_{1}, R_{2}, \ldots$ are all the rules form the knowledge base $K$, and for each rule $R$ of the type $P_{1}\left(x_{i_{1}}\right), \ldots, P_{m}\left(x_{i_{m}}\right) \rightarrow P(u)$, we define $\pi_{R}$ as $\pi_{R}=f_{\&}\left(P_{1}\left(x_{i_{1}}\right), \ldots, P_{m}\left(x_{i_{m}}\right), P(u)\right)$. Likewise, $\tilde{\pi}_{C}(u)=\tilde{f}_{v}\left(\bar{\pi}_{R_{1}}, \tilde{\pi}_{R_{2}}, \ldots\right)$, where $f_{v}(a, b)=\min (a+b, 1)$, and $\tilde{\pi}_{R}=\min \left(P_{1}\left(x_{i_{1}}\right), \ldots, P_{m}\left(x_{i_{m}}, P(u)\right)\right.$.

$1^{\circ}$. According to our Definition 4 (of an and-or pair), $f_{\& \varepsilon}(a, b) \leq \min (a, b)$ for all $a, b$. Therefore, for each rule $R_{k}, \min \left(P_{1}\left(x_{i_{1}}\right), \ldots, P_{m}\left(x_{i_{m}}\right), P(u)\right) \geq f_{k}\left(P_{1}\left(x_{i_{1}}\right), \ldots, P_{m}\left(x_{i_{m}}\right), P(u)\right)$, i.e., $\tilde{\pi}_{R_{k}} \geq \pi_{R_{k}}$.

$2^{\circ}$. According to Definition $4, f_{V}$ if non- decreasing in each of the variables. So, from $\bar{\pi}_{R_{k}} \geq \pi_{R_{k}}$, we conclude that $\pi_{C}(u)=f_{V}\left(\pi_{R_{1}}, \pi_{R_{2}}, \ldots\right) \leq f_{V}\left(\tilde{\pi}_{R_{1}}, \tilde{\pi}_{R_{2}}, \ldots\right)$.

$3^{\circ}$. Now, according to Definition $4, f_{v}(a, b) \leq f_{v}(a, b)$ for all $a, b$. Hence, $f_{v}\left(\tilde{\pi}_{R_{1}}, \tilde{\pi}_{R_{3}}, \ldots\right) \leq$ $\bar{f}_{V}\left(\tilde{\pi}_{R_{1}}, \bar{\pi}_{R_{2}}, \ldots\right)=\bar{\pi}_{C}(u)$.

Combining $2^{\circ}$ and $3^{\circ}$, we conclude that $\pi_{C}(u) \leq \pi_{C}(u)$. Application of the Lemma completes the proof.

Proof of Theorem 2. Here, $\pi_{C}(u)=f_{V}\left(\pi_{R_{1}}, \pi_{R_{2}}, \ldots\right)$, where and for each rule $R$ of the type $P_{1}\left(x_{i_{1}}\right), \ldots, P_{m}\left(x_{i_{m}}\right) \rightarrow P(u), \pi_{R}=f_{\&}\left(P_{1}\left(x_{i_{1}}\right), \ldots, P_{m}\left(x_{i_{m}}\right), P(u)\right)$. Likewise, $\tilde{\pi}_{C}(u)=\max \left(\tilde{\pi}_{R_{1}}, \tilde{\pi}_{R_{2}}, \ldots\right)$, where $\tilde{\pi}_{R}=\tilde{f}_{\&}\left(P_{1}\left(x_{i_{1}}\right), \ldots, P_{m}\left(x_{i_{m}}\right), P(u)\right)$ and $\tilde{f}_{\&}(a, b)=a b$.

$1^{\circ}$. According to our definition of a correlated and-or pair, $f_{k}(a, b) \geq a b=\tilde{f}_{\&}(a, b)$ for all $a, b$. Therefore, for every rule $R_{k}, f_{k}\left(P_{1}\left(x_{i_{1}}\right), \ldots, P_{m}\left(x_{i_{m}}\right), P(u)\right) \geq \tilde{f}_{k}\left(P_{1}\left(x_{i_{1}}\right), \ldots, P_{m}\left(x_{i_{m}}\right), P(u)\right)$, i.e., $\pi_{R_{k}} \geq \tilde{\pi}_{R_{k}}$.

$2^{\circ}$. According to Definition 4, $f_{V}$ if non-decreasing in each of the variables. So, from $\pi_{R_{k}} \geq \tilde{\pi}_{R_{k}}$, we conclude that $\pi_{C}(u)=f_{V}\left(\pi_{R_{1}}, \pi_{R_{2}}, \ldots\right) \geq f_{V}\left(\tilde{\pi}_{R_{1}}, \bar{\pi}_{R_{2}}, \ldots\right)$.

$3^{\circ}$. Now, according to Definition $4, f_{V}(a, b) \geq \max (a, b)$ for all $a, b$. Hence, $f_{V}\left(\tilde{\pi}_{R_{1}}, \tilde{\pi}_{R_{2}}, \ldots\right) \geq$ $\max \left(\tilde{\pi}_{R_{1}}, \bar{\pi}_{R_{2}}, \ldots\right)=\bar{\pi}_{C}(u)$.

Combining $2^{\circ}$ and $3^{\circ}$, we conclude that $\pi_{C}(u) \geq \bar{\pi}_{C}(u)$. Application of the Lemma completes the proof.

ACKNOWLEDGEMENTS. This work was supported by NSF Grant No. CDA - 9015006, NASA Research Grant No. 9-482 and the Institute for Manufacturing and Materials Management grant. The authors are thankful to Bob Lea, Ron Yager, and John Yen for encouraging discussions.

\section{REFERENCES}

1. Chang, S. and Zadeh, L.(1972). On Fuzzy Mapping and Control, IEEE Trensactions on Systems, Man and Cybernetics, Vol. SMC-2.

2. Cheeseman, P.(1983). In Defense of Probability, Proceedings of the 8-th International Joint Conference on $A I$, Los Angeles.

3. Nguyen, H. and Kreinovich, V.(1992). Maximum Entropy Approach to Fuzzy Control, University of Texas at El Paso Technical Report UTEP-CS-92-16a.

4. Jaynes, E.(1979). Where Do We Stand on Maximum Entropy? Levine, R. and Tribus, M. (Eds.) The maximum Entropy Formalism, MIT Press, Cambridge, MA.

5. Klir, G. and Folger, T.(1988). Fuzzy Sets, Uncertainty and Information. Prentice Hall, Englewood Cliffs, NJ.

6. Kosheleva, O. and Kreinovich, V.(1979). A letter on maximum entropy method. Nature, Vol. 281.

7. Kreinovich, V.(1989). Entropy Approach for the Description of Uncertainty in Knowledge Bases, Technical Report, Center for the New Informational Technology "Informatika" (in Russian).

8. Kreinovich, V., Quintana, C. and Lea, R.(1991). What Procedure to Choose While Designing a Fuzzy Control? Towards Mathematical Foundations of Fuzzy Control, Working Notes of the 1st International Workshop on Industrial Applications of Fuzzy Control and Intelligent Systems, College Station, TX.

9. Kreinovich, V. et al.(1992). What Non-linearity to Choose? Mathematical Foundations of Fuzzy Control. Proceedings, International Fuzzy Systems and Intelligent Control Conference, Louisville, KY.

10. Lee, C.(1990). Fuzzy Logic in Control Systems: Fuzzy Logic Controller. IEEE Transactions on Systems, Man and Cybernetics, Vol. 20, No. 2.

11. Mamdani, E.(1974). Application of Fuzzy Algorithms for Control of Simple Dynamic Plant, Proceedings of the IEE, Vol. 121, No. 12.

12. Ramer, A.(1990). Concepts of Fuzzy Information Measures On Continuous Domains, Int. J. General Systems, Vol. 17.

13. Ramer, A. and Yager, R.(1992). Analysis of Specificity of Fuzzy Sets, Proceedings of the IEEE International Conference on Fuzzy Systems, San Diego, CA.

14. Yen, Y., Pfluger, N. and Langari, R.(1992). A Defuzzification Strategy For a Fuzzy Logic Controller Employing Prohibitive Information in Command Formulation, Proceedings of IEEE International Conference on Fuzzy Systems, San Diego, CA.

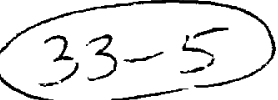

